\title{
Circadian regulation of light-evoked attraction/avoidance in day- vs. night-biting mosquitoes
}

Lisa Soyeon Baik ${ }^{1}$, Ceazar Nave ${ }^{1}$, David D. Au ${ }^{1}$, Tom Guda ${ }^{2}$, Joshua A. Chevez ${ }^{1}$, Anandasankar Ray $^{2}$, and Todd C. Holmes ${ }^{1 \S}$

${ }^{1}$ Department of Physiology and Biophysics, School of Medicine, University of California at Irvine, Irvine, California, United States of America

${ }^{2}$ Department of Molecular, Cell and Systems Biology, University of California Riverside, Riverside, California, United States of America.

Correspondence: ${ }^{\S}$ E-mail: tholmes@uci.edu, Telephone: 949-824-0006 


\begin{abstract}
Mosquitoes pose widespread threats to humans and other animals as disease vectors. Day- vs. night-biting mosquitoes occupy distinct time-of-day niches and exhibit very different innate temporal attraction/avoidance behavioral responses to light, yet little is known about their circuit or molecular mechanisms. Day-biting diurnal mosquitoes Aedes aegypti are attracted to light during the day regardless of spectra. In contrast, night-biting nocturnal mosquitoes Anopheles coluzzii avoid short, but not long wavelength light. Attraction/avoidance behavioral responses to light in both species change with time-of-day and show distinct sex and circuit differences. The basis of diurnal versus nocturnal behavior is driven by clock timing, which cycle anti-phase between day-biting versus night-biting mosquito species. Disruption of the circadian molecular clock severely interferes with light-evoked attraction/avoidance behavior in mosquitoes. In summary, attraction/avoidance mosquito behaviors are circadian and light regulated, which may be applied towards species specific control of harmful mosquitoes.
\end{abstract}

\title{
Introduction
}

Mosquitoes present a worldwide threat as key disease vectors that spread malaria parasites and Zika, Chikungunya, West Nile, Yellow Fever, and Dengue Fever viruses. Toxic pesticides are environmentally costly in contrast to light-based insect control approaches. However, light-based insect control approaches do not typically take into consideration the dayvs. night behavioral activity profiles of insects. Insects display a wide range of short wavelength light modulated behaviors, including attraction/avoidance ${ }^{1-7}$. It has been long assumed that insect responses to ultraviolet (UV) light are mediated by UV-sensitive opsins expressed in eyes and other external photoreceptors. Mosquitoes and flies also express non-opsin photoreceptors including the blue-light sensitive flavoprotein CRYPTOCHROME (CRY) ${ }^{8}$ which mediates a wide range of behavioral responses to blue and UV light, including circadian modulated attraction and avoidance ${ }^{1,2}$. Different mosquito species have evolved distinct circadian timing of behaviors according to their temporal/ecological niches, which likely minimize inter-species competition. Some mosquito species are diurnal (i.e., Aedes aegypti) while others are nocturnal (i.e., Anopheles coluzzii). Numerous mosquito behaviors change with the time-of-day, including flight activity, mating, oviposition, and biting ${ }^{9-15}$. Considering their impact on health and ecology, little is known about the species basis of diurnality/nocturnality and behavioral timing in mosquitoes.

\section{Results}

We measured the light environment preference behaviors of diurnal (Aedes aegypti) and nocturnal (Anopheles coluzzii) mosquito species throughout the $24 \mathrm{hr}$ day using a custom designed arena (S1 Fig.). Young adult mosquitoes were presented with a choice of light versus shaded environments during the subjective daytime (ZT 0-12) to measure preference for the light-exposed or in the shaded-environment, quantified as $\%$ of preference. Nocturnal versus diurnal mosquito species exhibit striking differences in their light-evoked attraction/avoidance behavior, despite constant light intensity. Diurnal Aedes aegypti (Ae. aegypti) females are 
behaviorally attracted to UV light during the day (Fig. 1A and 1J). In contrast, nocturnal Anopheles coluzzii (An. coluzzii) females strongly avoid UV light during most of the daytime (Fig. 1B and 1J). Both Ae. aegypti and An. coluzzii females show shifted attraction/avoidance behavior as dusk approaches even under constant light intensity until lights off (Fig. 1A, 1B, 1E, and $1 \mathrm{G})$. This temporal "anticipatory" afternoon behavioral shift starts much earlier for diurnal Ae. aegypti females, which increase their attraction behavior beginning around mid-afternoon (ZT6) (Fig. 1A and 1E). In contrast, nocturnal An. coluzzii, females show sharp decreases in UV light avoidance starting before dusk (ZT11) (Fig. 1G). Precisely at dusk, they enter the previously UV illuminated area, rapidly reaching 60\% preference for this area 10 minutes after dusk and peak $70 \%$ preference one hour after dusk (Fig. 1B).

Male mosquitoes form swarms in anticipation of females, thus we considered the possibility of sex differences for avoidance/attraction behavioral responses to UV light for both diurnal and nocturnal mosquito species. Diurnal Ae. aegypti males are attracted to UV light during the late subjective daytime, but to a significantly lesser extent than females, which are attracted to UV light throughout the entire day (Fig. 1C and 1I). Nocturnal An. coluzzii males strongly avoid UV light, similar to An. coluzzii females (Fig. 1D and 1I). Both species show sexspecific differences in timing of "anticipation" of increase in attraction/decrease in avoidance while temporally approaching dusk. Ae. aegypti male attraction peaks much earlier (ZT10) than that of females (ZT 12) (Fig. 1A, 1C, 1E, and 1F). Similarly as dusk approaches, An. coluzzii males initiate decreases in avoidance much earlier than females during their transition to attraction to the previously UV light illuminated area (Fig. 1B, 1D, 1G, and 1H). Sex-dependent differences persist even after the UV light is turned off, simulating the subjective nighttime (ZT 12-24). For both species, females prefer the previously light-exposed environment throughout the night. This nighttime preference for previously UV-light exposed environment is significantly higher in females, compared to males (Fig. 1A-1D, and 1J).

The color spectral specificity of attraction/avoidance behavior varies between different insect species ${ }^{1,2,4,5,16}$. Drosophila melanogaster diptera fruit flies avoid short wavelength light, but not long wavelength light and Drosophila UV light avoidance peaks in the midday during their low locomotor activity "siesta"1,2. To determine the spectral dependence of mosquito attraction/avoidance behavioral light responses, we compared their environmental preferences for visible short wavelength blue light and visible long wavelength red light for comparison with UV light (Fig. 1). Diurnal Ae. aegypti females are attracted to both blue and red light during the daytime, very similar to their behavioral attraction to UV light (Fig. 1A, and Panels A, C, and E in S2 Fig.). Ae. aegypti females are equally attracted to all light wavelengths tested (Panel E in S2 Fig.). Nocturnal An. coluzzii females, which strongly avoid UV light (Fig. 1B), also avoid blue light during the day (Panel B in S2 Fig.). Their magnitude of blue light avoidance is significantly lower than their UV light avoidance (Panel E in S2 Fig.). In contrast to their striking short wavelength avoidance during the day, female An. coluzzii are slightly attracted to long wavelength red light (Panels D and E in S2 Fig.). During the nighttime, females of both species prefer environments with prior UV light-exposure (Fig. 1J and Panel F in S2 Fig.), significantly higher than their nighttime preference for areas with prior blue- or red-light exposure, for which they are weakly attracted to (Panel F in S2 Fig.). Thus, attraction/avoidance behavioral responses are wavelength-dependent and differ in both overall profile and anticipation of dusk between nocturnal and diurnal species. 
We anatomically mapped the circadian neuronal network in the central brain of female diurnal and nocturnal mosquitoes, motivated by the apparent relationship between the circadian clock and circuit modulation of light attraction/avoidance behavior, defined by the cyclic expression of PERIOD (PER) clock protein that drives rhythmic changes in physiology and behavior. In Drosophila melanogaster, Pigment Dispersing Factor (PDF) is a neuropeptide coexpressed with PER in the small- and large-lateral ventral neurons (LNvs), which modulate circadian- and light-mediated behaviors such as circadian locomotion, sleep, arousal, and lightevoked attraction/avoidance behaviors ${ }^{1,2,17-20}$. PER and PDF proteins are co-expressed in a similar neuroanatomical pattern the lateral ventral area in both Ae. aegypti and An. coluzzii female adult brains and are anatomically similar to Drosophila melanogaster and some other insects ${ }^{21,22}$. These $\mathrm{PDF}^{+}$and $\mathrm{PER}^{+}$neurons can be further distinguished by size, location and projections as large- (1-LNvs) and small-lateral ventral neurons (s-LNvs) in Ae. aegypti and An. coluzzii (Fig. 2 and S3 Fig.) and feature large neuronal arbors in the optic lobes dorsal projections, similar to Drosophila ${ }^{20}$ (S4 Fig., S1 Movie, and S2 Movie). The neuroanatomical locations of $\mathrm{PER}^{+}$neurons show species specific similarities and differences for cell groups between diurnal Ae. aegypti and nocturnal An. coluzzii outside of the lateral ventral area. Similarly located neuronal groups include putative dorsal neurons (DNs) in Ae. aegypti and An. coluzzii (Fig. 2 and S3 Fig.). Differentially located neuronal groups include approximately 5 $\mathrm{PER}^{+}$neurons in the medial-anterior region of Ae. aegypti female brains, which we call medialanterior neurons (m-ANs) here (Fig. 2 and S3 Fig.). Based on location and size, another differentially located neuronal group include approximately $7 \mathrm{PER}^{+} / \mathrm{PDF}^{-}$neurons, which resemble the pars intercerebralis (PI) in An. Coluzzii (Fig. 2). In Drosophila melanogaster, PI neurons are physiologically circadian rhythmic although by way of other clock neuron input ${ }^{23,24}$. These PI-like PER ${ }^{+} / \mathrm{PDF}^{-}$neurons are not detected in Ae. aegypt (Fig. 2 and S3 Fig.). In summary, we observe both shared and distinct anatomical features of circadian neuronal circuit of diurnal versus nocturnal mosquito species.

We measured PER protein oscillating levels throughout the $24 \mathrm{hr}$ day using anti-PER immunocytochemistry staining, co-stained with PDF, at $6 \mathrm{hr}$ intervals in the brains of standard 12hr: 12hr light:dark (LD) entrained Ae. aegypti and An. coluzzii female mosquitoes. PER rhythms cycle robustly in both Ae. aegypti and An. coluzzii circadian neurons in a neuronal subgroup-specific manner. Notably, PER cycling in $\mathrm{PDF}^{+}$LNvs oscillate in opposite phases between diurnal Ae. aegypti versus nocturnal An. coluzzii brains (Fig. 3). PER protein levels peak in late night/early day in $\mathrm{PDF}^{+}$s-LNv and l-LNv of the diurnal mosquito Ae aegypti (Fig. 3A, $3 \mathrm{C}$, and 3D). In contrast, PER protein levels peak in late day/early night in $\mathrm{PDF}^{+} \mathrm{s}-\mathrm{LNv}$ and $1-$ LNv of the nocturnal mosquito An. coluzzii (Fig. 3B, 3E, and 3F). PER protein levels peak in early daytime in $\mathrm{PER}^{+}$DNs of both diurnal Ae. aegypti and nocturnal An. coluzzii (S5 Fig. and S7 Fig.). Similarly, PER protein levels peak during the daytime in Ae. aegypti specific m-ANs (S6 Fig.). In An. coluzzii PI-like neurons, PER protein levels peak in the early day (S8 Fig.). In summary, diurnal and nocturnal mosquitoes have distinct circadian molecular signatures in the brain, with an early day PER peak in diurnal mosquito Ae. aegypti versus an early evening PER peak in nocturnal An. coluzzii in $\mathrm{PDF}^{+} \mathrm{LNv}$ neurons. These opposing oscillation phases suggest a mechanism for diurnal and nocturnality. 
Constant light condition disrupts circadian clock gene expression and rhythmic behaviors in many animals, including mosquitoes ${ }^{10,11,25}$. To examine the functional linkage between circadian clock disruption and attraction/avoidance behavioral responses to UV light, Ae. aegypti and An. coluzzii female mosquitoes were exposed to constant UV light exposure (UV LL) for 3-5 days. Using anti-PER immunocytochemistry, we measured PER protein levels corresponding to species specific peak times in female mosquito brains following 3-5 days of UV LL. PER protein levels are severely reduced in mosquito brains following UV LL compared to LD in both Ae. aegypti and An. coluzzii (Fig. 4A-4D). In many brains, PER protein levels in LNvs could not be quantified because there was no visible PER staining following UV LL (not shown). We then measured the behavioral preference for UV-exposed versus shaded light environments under UV LL condition. During UV LL condition, both Ae. aegypti and An. coluzzii mosquitoes lack clear time-of-day dependent changes in attraction/avoidance behavior, including the anticipation (Fig. 4E-4H). Ae. aegypti females are attracted to UV light regardless of time-of-day (Fig. 4E and 4G). An. coluzzii females lack subjective day versus night differences in avoidance/attraction, and overall lack any clear preferences for either UV-exposed or shaded environments under UV LL condition (Fig. 4F and 4H). Clock ablation severely disrupts the timing of UV-evoked attraction/avoidance behavior of both diurnal and nocturnal mosquitoes.

\section{Discussion}

We show that mosquito attraction/avoidance behavior to light is specie-, sex-, spectra-, and time-of-day-dependent. Diurnal versus nocturnal mosquitoes have opposite attraction/avoidance behavioral valence for short wavelength light. Daytime-active mosquitoes, Ae. aegypti, are attracted to a wide range of light spectra during the daytime. In contrast nighttime-active mosquitoes An. coluzzii are strongly photophobic in response to short wavelength light. Both species exhibit anticipatory behavior of decreased avoidance and increased attraction during the temporal approach to dusk. This correlates with the ecological timing of increased flight activity and host seeking behaviors ${ }^{10,11}$. Interestingly, males of both diurnal and nocturnal species show earlier anticipatory behavior of increased UV attraction, compared to females. Both Ae. aegypti and An. coluzzii males exhibit earlier flight activity onset towards dusk, compared to females ${ }^{15,26}$. Male mosquitoes form 'swarms' in anticipation of female mosquitoes flying through to mate. The timing of male swarming is species specific. This dictates a temporal niche for mating of different mosquito species, whereby male mosquitoes evolved pre-dusk earlier anticipation to optimize their chance of mating ${ }^{12,15}$. Our previous work shows that light-evoked attraction/avoidance behavior is mediated by both opsinand non-opsin based photoreceptors in Drosophila ${ }^{1,2}$. Further investigation is needed to determine whether if different phototransduction mechanisms are involved in mosquito light preference behavior.

We show the location and oscillation of PER protein at neuronal subgroup level in diurnal and nocturnal mosquitoes. Intriguingly, we find PER protein expression oscillates in anti-phasic manner between diurnal and nocturnal mosquitoes, which could mechanistically underlie diurnal versus nocturnal behaviors. Conceptual support for this finding is that for diurnal versus nocturnal mammals, the circadian clock in non-suprachiasmatic nuclei neurons and periphery tissues cycle in opposite phases between diurnal versus nocturnal rodents and 
primates $^{27-29}$. It is not known currently what factors contribute to differential phase timing of circadian protein cycling among different tissue and cellular types. Our detailed characterization of light-evoked attraction/avoidance behavior in mosquitoes shows timing features that suggest that these processes are under circadian regulation as we find previously in Drosophila melanogaster $^{1,2}$. Experimental verification for this is shown by temporal disruption of lightevoked attraction/avoidance behavior by environmentally shutting down the circadian clock with constant light (LL). A wide range of behaviors in mosquito and other insects are temporally modulated by light, including mating, seeking a blood-meal, biting, oviposition, flight activity, and sleep ${ }^{9-15}$. Light treatments that alter circadian function also disrupts biting, flight activity, and oviposition behaviors $6,7,10,11,14$. By controlling the timing and color of light exposure, we can further specify mosquito species being targeted towards specific manipulation of mosquito behaviors using environmentally friendly light-based approaches.

\section{Materials and Methods}

\section{Immunocytochemistry}

All mosquitoes were reared in standard $12 \mathrm{hr}: 12 \mathrm{hr}$ light: dark (LD) schedule at $27^{\circ} \mathrm{C}$, and $80 \%$ humidity in large cages, with access to $10 \%$ sucrose diet. Mosquito brains were dissected 5-10 days post-eclosion. Brains were dissected in $1 \mathrm{X}$ PBS, fixed in $4 \%$ paraformaldehyde (PFA) for $30 \mathrm{~min}$, washed $3 \mathrm{X} 10 \mathrm{~min}$ in PBS-Triton-X 1\%, incubated in blocking buffer $(10 \%$ Horse Serum-PBS-Triton-X $0.5 \%)$ at room temperature before incubation with mouse $\alpha$-PDF C7, monoclonal $(1: 10,000)$ and rabbit $\alpha$-PER, polyclonal $(1: 1,000)$ antibodies overnight in $4^{\circ} \mathrm{C}$. Primary antibody incubated brains were then washed $3 \mathrm{X} 10 \mathrm{~min}$ in PBSTriton-X $0.5 \%$ then incubated in goat $\alpha$-mouse-Alexa- (1:500) and goat $\alpha$-rabbit-Alexa-594 (1:500) secondary antibodies in blocking buffer overnight in $4^{\circ} \mathrm{C}$. Brains were washed $5 \mathrm{X}$ $15 \mathrm{~min}$ in PBS-Triton-X $0.5 \%$ before mounting in Vectashield mounting media (Vector Laboratories). Microscopy was performed using Zeiss LSM700 confocal microscope. Fluorescence levels were analyzed using Imaris software (Bitplane). Spherical region of interest was selected for each cell and fluorescence was quantified for each region by the Imaris software. Each species timepont were collected for minimum of three repetitions each. Reported quantification values reflect the average fluorescence intensity levels and error bars indicate S.E.M.

\section{Light-Induced Attraction/Avoidance Behavioral Assay}

All mosquitoes were reared in standard $12 \mathrm{hr}: 12 \mathrm{hr}$ light: dark (LD) schedule in $27^{\circ} \mathrm{C}$, and $80 \%$ humidity in large cages, with access to $10 \%$ sucrose diet. Adult mosquitoes (0-5 days post-eclosion) were entrained to LD schedule for minimum of 3 days prior to testing. Individual mosquitoes were each placed into $25 \mathrm{~mm}$ diameter x $125 \mathrm{~mm}$ length pyrex glass tubes (Trikinetics) plugged with "flugs" on either side. Flugs are soaked with $10 \%$ sucrose providing a food source, while simultaneously allowing airflow sufficient for multi-day survival of the mosquitoes in the tubes. Tubes containing individual mosquitoes were placed in humidity-, temperature-, and light-controlled incubator and allowed to acclimate for a full day. One half of the tubes were covered with infrared (IR) filters (LEE Filters 4 x 4" Infrared (87C) Polyester Filter), providing the mosquitoes with a choice of a shaded environment (IR filtered) versus 
light-exposed (not covered with IR filter) during the $12 \mathrm{hrs}$ of light. Philips TL-D Blacklight ultraviolet light (UV) source with narrow peak wavelength of $365 \mathrm{~nm}$ and intensity of 400 $\mu \mathrm{W} / \mathrm{cm}^{2}$ was used for UV light. Blue (450 nm, Supernight) and red (630 nm, Supernight) LED strips set around $400 \mu \mathrm{W} / \mathrm{cm}^{2}$ was used as blue and red light sources. Additionally, IR LED strips (Infrared $850 \mathrm{~nm} 3528$ LED Strip Light, 78/m, $8 \mathrm{~mm}$ wide, by the $5 \mathrm{~m}$ Reel) placed on aluminum heat sink was placed under the entire setup. With each light source, same LD schedule as the LD entrainment schedule prior to experiment was continued to minimize any disturbance to the circadian time. For constant light (LL) light choice assay, the UV light was constantly left on, instead of LD. Webcam (Microsoft Q2F-00013 USB 2.0 LifeCam) took pictures at 5min intervals for 3-5 days of experiment. Each mosquito's preference in the light-exposed versus shaded side of the tube was analyzed by the ImageJ program. Each experiment was repeated a minimum of three repetition for each group. Preference was averaged for each time point per mosquito and statistical measurements were analyzed by t-test using Microsoft Excel and Sigma Plot.

\section{Acknowledgments}

We thank Annika Barber, Naoki Okamoto, and Anupama Dahanukar for helpful discussions; Guiyun Yan and Adeela Syed for technical support; and Janita Parpana, Eleanor Chan, Duke Park, and Lillian Li for administrative support. TCH is supported by R35 GM127102. LSB acknowledges partial support by an individual NSF pre-doctoral fellowship award. A.R. is founder of Sensorygen Inc, a startup working on natural odorants as insect repellents and food flavorings.

\section{Author Contributions}

L.S.B. conceptualized, designed, acquired, analyzed, interpreted, and drafted this work; C.N. acquired and analyzed data; D.A. analyzed data; T.G. contributed to designing this work; J.A.C. acquired data; A.R. contributed to designing, interpreting, and revising this work; T.C.H. contributed to interpreting and revising this work. 


\section{References}

1 Baik, L. S. et al. CRYPTOCHROME mediates behavioral executive choice in response to UV light. Proc Natl Acad Sci U S A 114, 776-781, doi:10.1073/pnas.1607989114 (2017).

2 Baik, L. S., Recinos, Y., Chevez, J. A. \& Holmes, T. C. Circadian modulation of lightevoked avoidance/attraction behavior in Drosophila. PLoS One 13, e0201927 (2018).

$3 \quad$ Knop, E. et al. Artificial light at night as a new threat to pollination. Nature 548, 206209, doi:10.1038/nature23288 (2017).

4 Tokushima, Y. et al. Broadband Photoreceptors Are Involved in Violet Light Preference in the Parasitoid Fly Exorista Japonica. PLoS One 11, e0160441, doi:10.1371/journal.pone.0160441 (2016).

5 Green, C. \& Cosens, D. Spectral responses of the tsetse fly, Glossina morsitans morsitans. J Insect Physiol 29, 795-800 (1983).

6 Farnesi, L. C., Barbosa, C. S., Araripe, L. O. \& Bruno, R. V. The influence of a light and dark cycle on the egg laying activity of Aedes aegypti (Linnaeus, 1762)(Diptera: Culicidae). Mem Inst Oswaldo Cruz 113 (2018).

7 Sheppard, A. D. et al. Light manipulation of mosquito behaviour: acute and sustained photic suppression of biting activity in the Anopheles gambiae malaria mosquito. Parasites \& Vectors 10, 255 (2017).

8 Liu, B., Liu, H., Zhong, D. \& Lin, C. Searching for a photocycle of the cryptochrome photoreceptors. Curr Opin Plant Biol 13, 578-586, doi:10.1016/j.pbi.2010.09.005 (2010).

9 Sumba, L. A. et al. Daily oviposition patterns of the African malaria mosquito Anopheles gambiae Giles (Diptera: Culicidae) on different types of aqueous substrates. J Circadian Rhythms 2, 6 (2004).

10 Jones, M. D., Hill, M. \& Hope, A. M. The circadian flight activity of the mosquito Anopheles Gambiae: phase setting by the light regime. J Exp Biol 47, 503-511 (1967).

11 Taylor, B. \& Jones, M. D. The circadian rhythm of flight activity in the mosquito Aedes aegypti (L.): the phase-setting effects of light-on and light-off. $J$ Exp Biol 51, 59-70 (1969).

12 Sawadogo, S. P. et al. Differences in timing of mating swarms in sympatric populations of Anopheles coluzzii and Anopheles gambiae s.s. (formerly An. gambiae M and S molecular forms) in Burkina Faso, West Africa. Parasit Vectors 6, 275, doi:10.1186/1756-3305-6-275 (2013).

13 Rund, S. S., Lee, S. J., Bush, B. R. \& Duffield, G. E. Strain-and sex-specific differences in daily flight activity and the circadian clock of Anopheles gambiae mosquitoes. J Insect Physiol 58, 1609-1619 (2012).

14 Das, S. \& Dimopoulos, G. Molecular analysis of photic inhibition of blood-feeding in Anopheles gambiae. BMC physiology 8, 23 (2008).

15 Araripe, L. O., Bezerra, J. R. A., Rivas, G. \& Bruno, R. V. Locomotor activity in males of Aedes aegypti can shift in response to females' presence. Parasit Vectors 11, 254, doi:10.1186/s13071-018-2635-9 (2018).

16 Coombe, P. Visual behaviour of the greenhouse whitefly, Trialeurodes vaporariorum. $J$ Phys Ent 7, 243-251 (1982).

17 Sheeba, V. et al. Large ventral lateral neurons modulate arousal and sleep in Drosophila. Curr Biol 18, 1537-1545, doi:10.1016/j.cub.2008.08.033 (2008). 
18 Parisky, K. M. et al. PDF cells are a GABA-responsive wake-promoting component of the Drosophila sleep circuit. Neuron 60, 672-682, doi:10.1016/j.neuron.2008.10.042 (2008).

19 Shang, Y., Griffith, L. C. \& Rosbash, M. Light-arousal and circadian photoreception circuits intersect at the large PDF cells of the Drosophila brain. Proc Natl Acad Sci US A 105, 19587-19594, doi:10.1073/pnas.0809577105 (2008).

20 Renn, S. C., Park, J. H., Rosbash, M., Hall, J. C. \& Taghert, P. H. A pdf neuropeptide gene mutation and ablation of PDF neurons each cause severe abnormalities of behavioral circadian rhythms in Drosophila. Cell 99, 791-802 (1999).

21 Helfrich-Forster, C., Nitabach, M. N. \& Holmes, T. C. Insect circadian clock outputs. Essays Biochem 49, 87-101, doi:10.1042/bse0490087 (2011).

22 Helfrich-Forster, C. The period clock gene is expressed in central nervous system neurons which also produce a neuropeptide that reveals the projections of circadian pacemaker cells within the brain of Drosophila melanogaster. Proc Natl Acad Sci U S A 92, 612-616 (1995).

23 Barber, A. F., Erion, R., Holmes, T. C. \& Sehgal, A. Circadian and feeding cues integrate to drive rhythms of physiology in Drosophila insulin-producing cells. Genes Dev 30, 2596-2606, doi:10.1101/gad.288258.116 (2016).

24 Cavanaugh, D. J. et al. Identification of a circadian output circuit for rest: activity rhythms in Drosophila. Cell 157, 689-701 (2014).

25 Konopka, R. J., Pittendrigh, C. \& Orr, D. Reciprocal behaviour associated with altered homeostasis and photosensitivity of Drosophila clock mutants. J Neurogenet 6, 1-10 (1989).

26 Rund, S. S., Lee, S. J., Bush, B. R. \& Duffield, G. E. Strain- and sex-specific differences in daily flight activity and the circadian clock of Anopheles gambiae mosquitoes. $J$ Insect Physiol 58, 1609-1619, doi:10.1016/j.jinsphys.2012.09.016 (2012).

27 Mure, L. S. et al. Diurnal transcriptome atlas of a primate across major neural and peripheral tissues. Science 359, eaao0318 (2018).

28 Lemos, D. R., Downs, J. L. \& Urbanski, H. F. Twenty-four-hour rhythmic gene expression in the rhesus macaque adrenal gland. Mol Endocr 20, 1164-1176 (2006).

29 Ramanathan, C., Nunez, A. \& Smale, L. Daily rhythms in PER1 within and beyond the suprachiasmatic nucleus of female grass rats (Arvicanthis niloticus). Neuroscience 156, 48-58 (2008). 


\section{Figure Legends}

Figure 1. UV light-evoked attraction/avoidance responses in diurnal and nocturnal mosquitoes are specie- and sex-dependent.

(a-d) Attraction/avoidance behavior to UV light, measured by \% of preference in UV-exposed versus shaded environment throughout $12 \mathrm{hr}$ : $12 \mathrm{hr}$ UV light: dark for female Ae. aegypti $(\mathrm{n}=110)$ (a), female An. coluzzii $(\mathrm{n}=88)$ (b), male Ae. aegypti $(\mathrm{n}=61)(\mathrm{c})$, male An. coluzzii $(\mathrm{n}=47)(\mathbf{d})$. (e-h) Attraction/avoidance behavior to UV light, measured by \% of preference in UV-exposed versus shaded-environment during ZT 6-12 for female Ae. aegypti (e), male Ae. aegypti (f), female An. coluzzii (g), male An. coluzzii (h). (i-j) Average attraction/avoidance behavioral preference to UV light-exposed versus shaded-environment for daytime (i) and nighttime (j) in Ae. aegypti and An. coluzzii female and male mosquitoes. Data are represented as mean \pm S.E.M. ${ }^{*} \mathrm{p}<0.05 ;{ }^{*} \mathrm{p}<0.01 ; * * * \mathrm{p}<0.001$ vs. female.

Figure 2. Schematic representation of Aedes aegypti and Anopheles coluzzii circadian neuronal circuits.

(a-b) Illustration of representative adult female central brains and its neurons expression PER and/or PDF. Asterisk (*) indicates groups distinct for each species. (a) Ae. aegypti s-LNv in color yellow, 1-LNv in color violet, DNs in color orange, and m-AN in color light blue, with $\mathrm{PDF}^{+}$neurons indicated with green outline. (b) An. coluzzii s-LNv in color yellow, $1-\mathrm{LNv}$ in color violet, DNs in color orange, and PI neurons in color dark blue, with $\mathrm{PDF}^{+}$neurons indicated with green outline.

Figure 3. Diurnal versus nocturnal mosquito PER expression in $\mathrm{PDF}^{+} \mathrm{LNv}$ neurons oscillate in anti-phasic manner

(a-b) Representative confocal images of adult female Ae. aegypti (a) and An. coluzzii (b) mosquito lateral ventral neurons (LNv) immunocytochemistry stained with $\alpha$-PER (magenta) and $\alpha-P D F$ (green) antibodies at ZTs 5, 11, 17, and 23. (c-f) PERIOD expression levels at each ZT for Ae. aegypti (ZT5, n=27; ZT11, n=17; ZT17, n=6, ZT23, n=7) s-LNv (c) and 1-LNv (d), and An. colluzzii (ZT5, n=13; ZT11, n=31; ZT17, n=9, ZT23, n=8) s-LNv (e) and 1-LNv (f). Data are represented as mean \pm S.E.M.

Figure 4. Constant UV light exposure disrupts circadian protein expression and clock modulation of attraction/avoidance behavioral responses to UV light in mosquitoes.

(a-b) Representative confocal images of anti-PER (magenta) and anti-PDF (green) immunocytochemistry stained adult female mosquito brains under LD or following UV LL exposure for Ae. aegypti (a) and An. coluzzii (b). (c-d) Average fluorescence intensity of circadian neurons under LD versus LL conditions for Ae. aegypti ZT/CT 23 (LD, n=7; LL, n=13) (c) and An. coluzzii ZT/CT 11 (LD, n=31; LL, n=6) (d). (e-f) Attraction/avoidance behavior to UV light, measured by $\%$ of preference in UV-exposed versus shaded during UV LL for female Ae. aegypti (e), female An. coluzzii (f). (g-h) Average attraction/avoidance behavioral preference to light-exposed versus shaded-environment during subjective daytime versus nighttime under LD or LL conditions for Ae. aegypti (g) and An. coluzzii (h) female mosquitoes. Data are represented as mean \pm S.E.M. ${ }^{*} \mathrm{p}<0.05 ; * * \mathrm{p}<0.01 ; * * * \mathrm{p}<0.001$ vs. LD or day. 

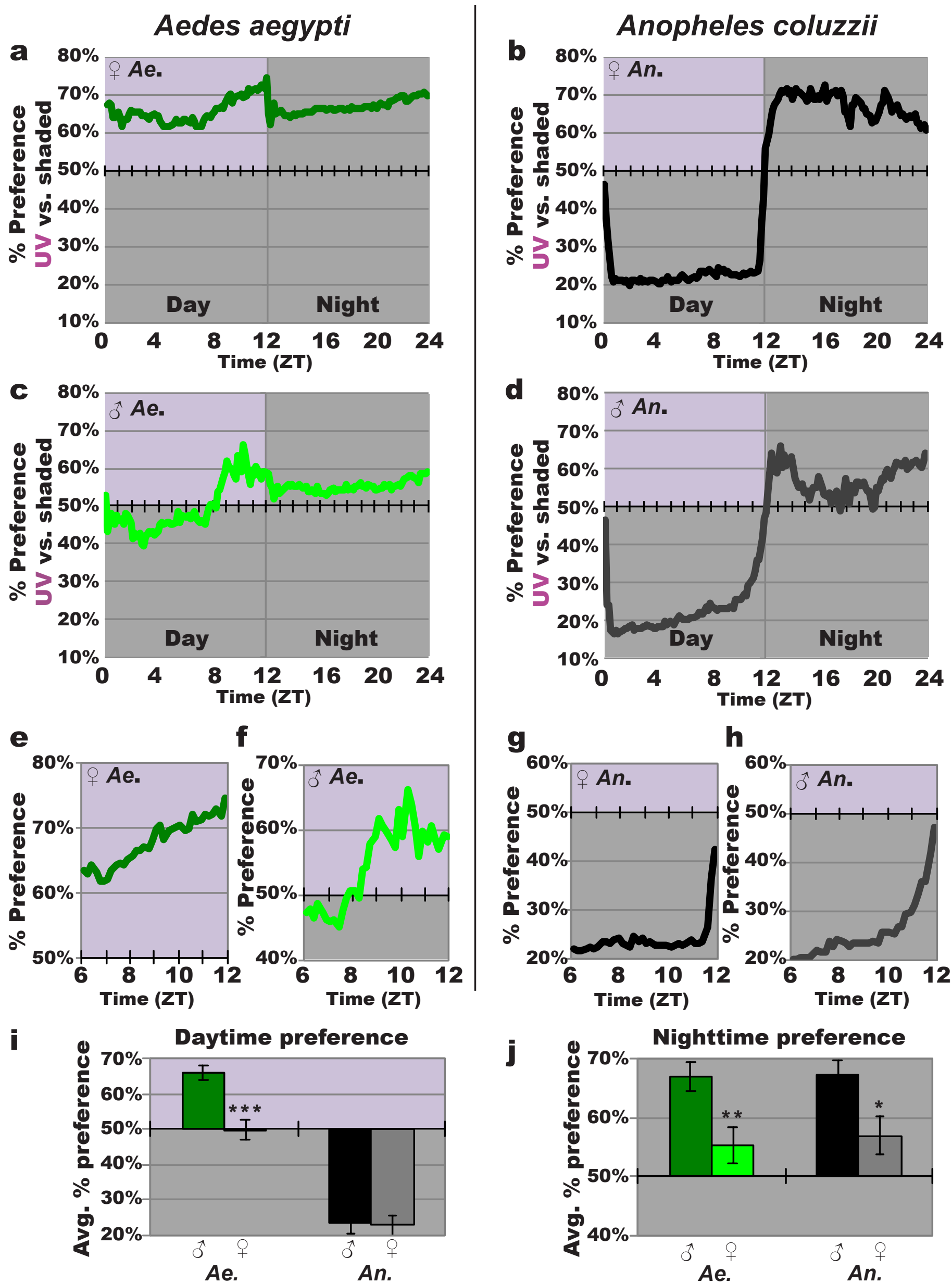


\section{Figure 2} certified by peer review) is the author/funder. All rights reserved. No reuse allowed without permission.

a

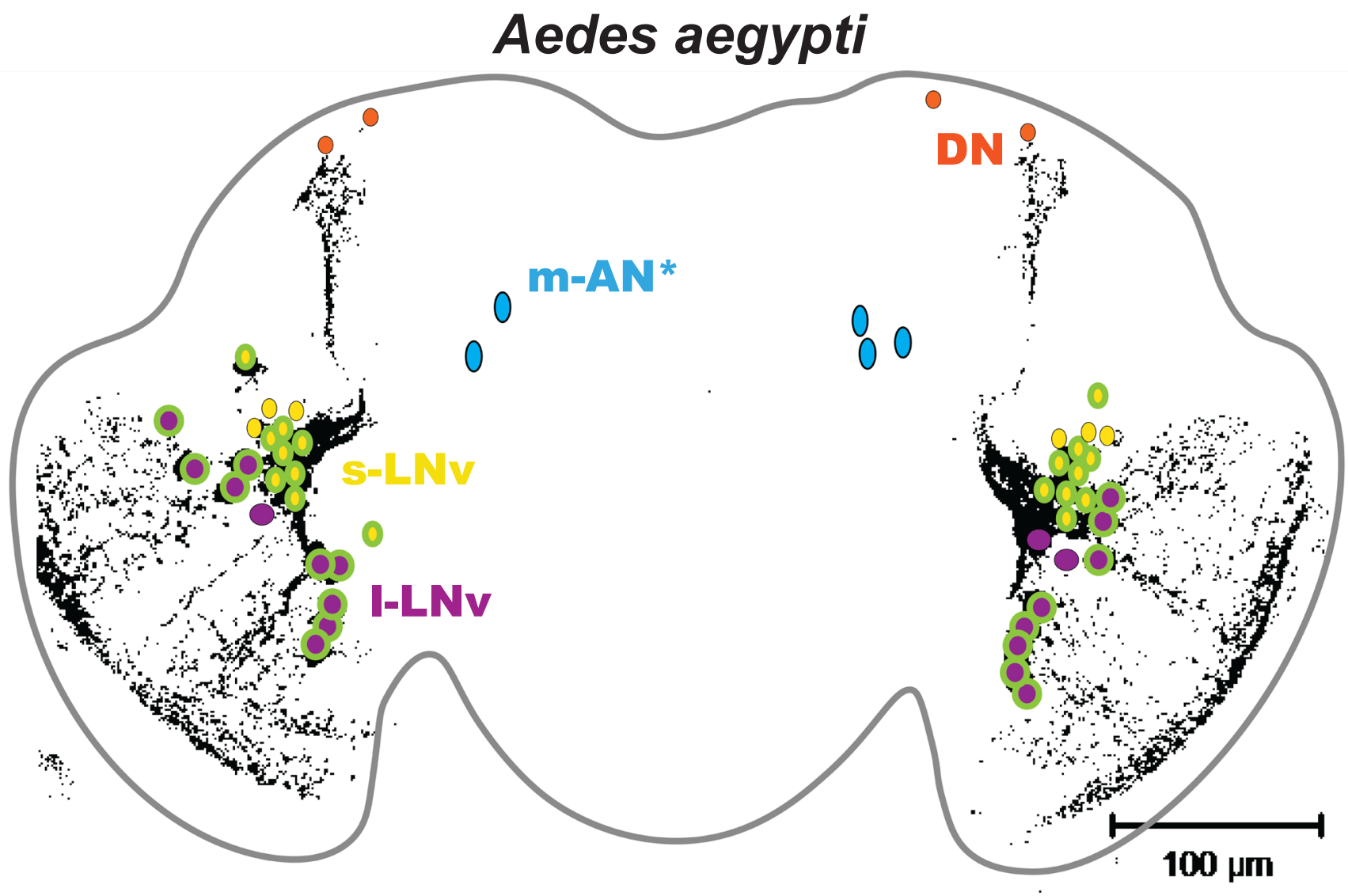

b

Anopheles coluzzii

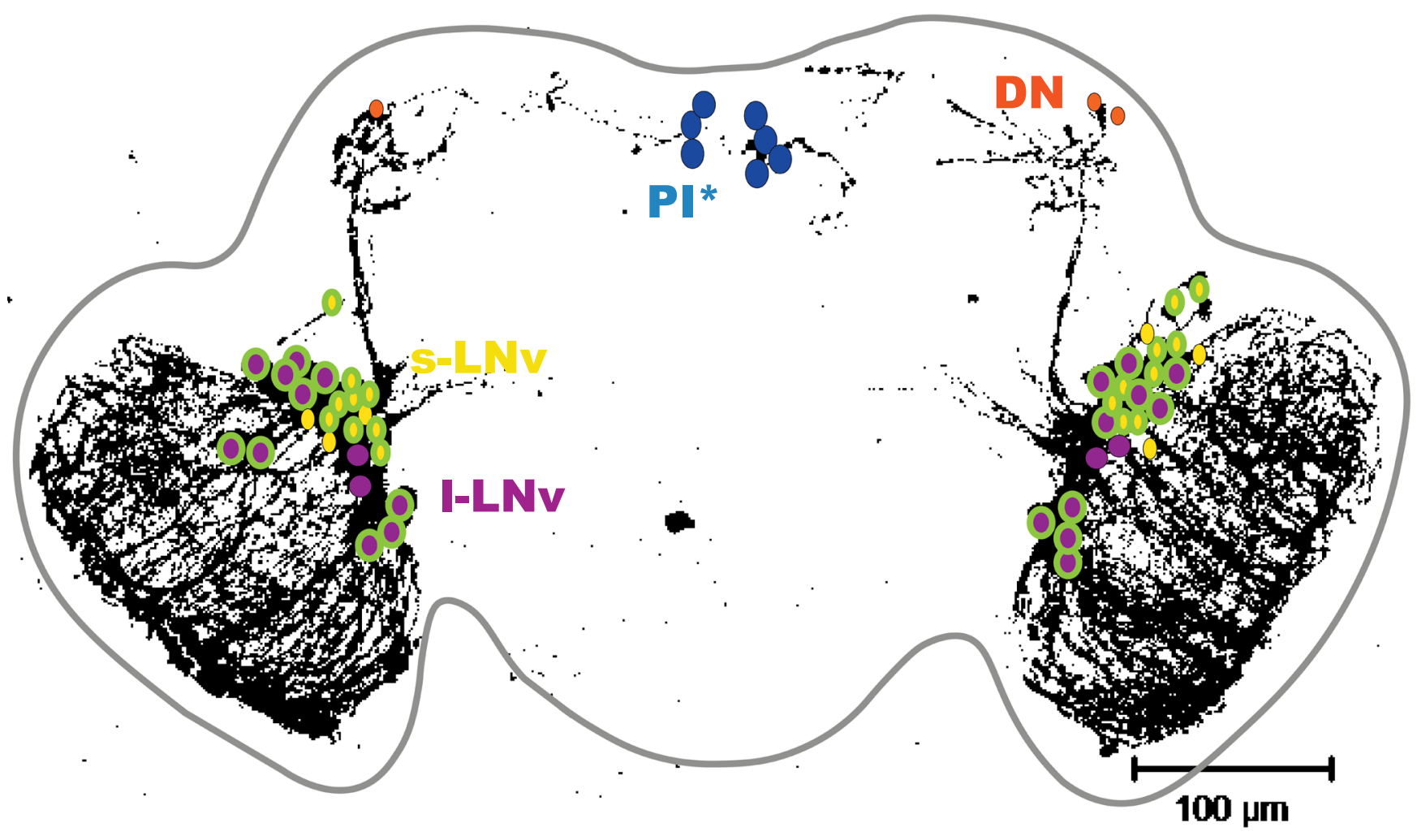


Figure 3
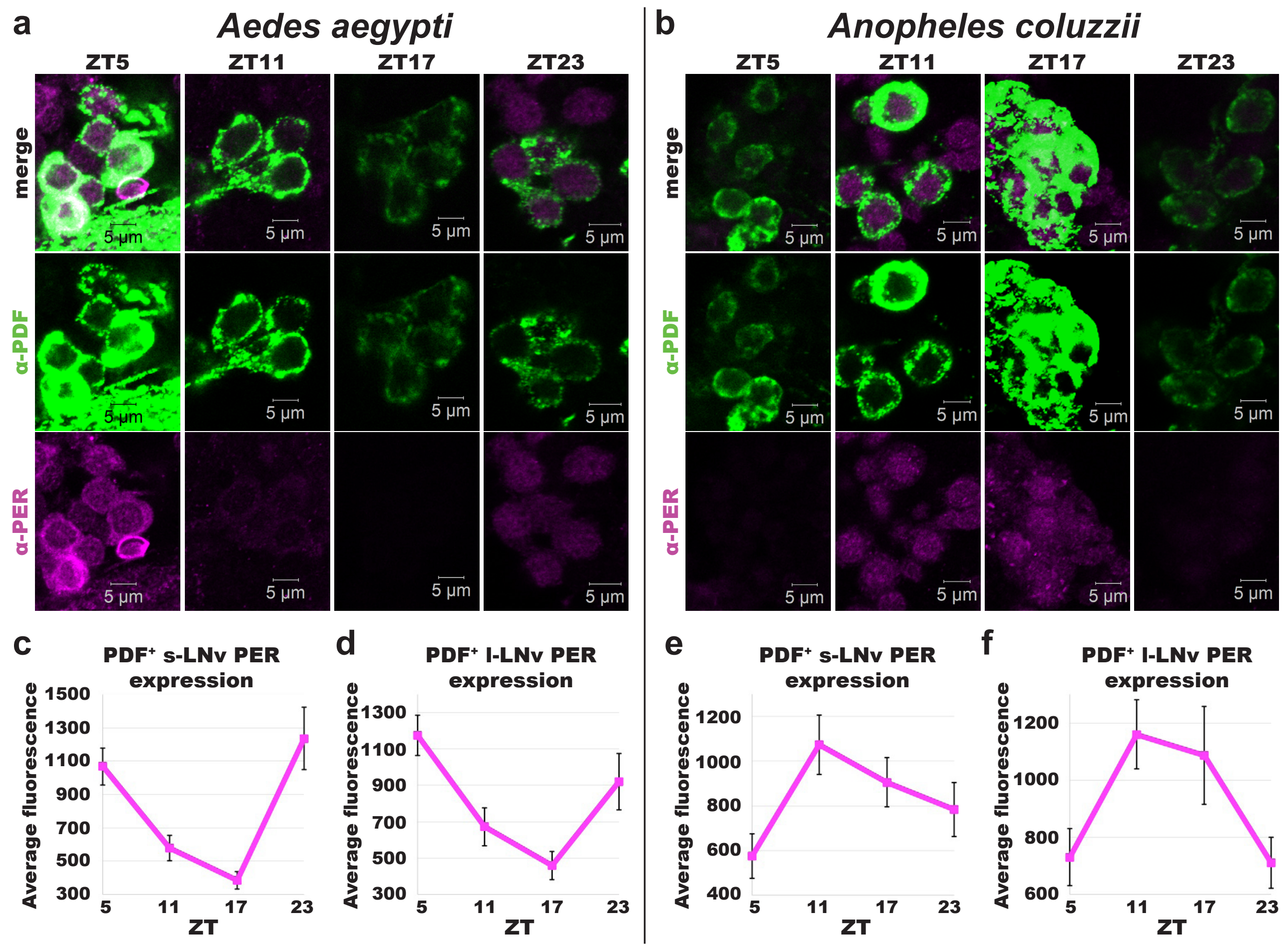
bioRxiv preprint doi: https://doi.org/10.1101/627588; this version posted May 5, 2019. The copyright holder for this preprint (which was not certified by peer review) is the author/funder. All rights reserved. No reuse allowed without permission.

a

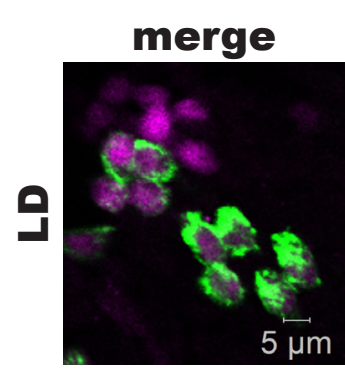

Aedes aegypti
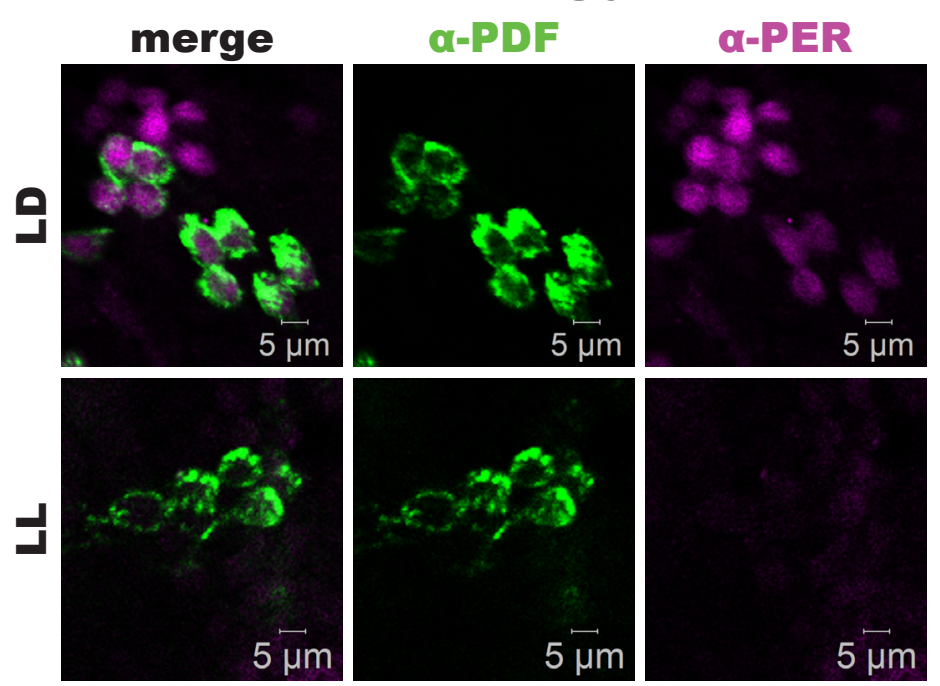

C

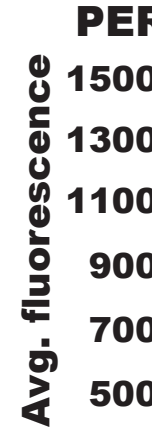

PER expression in LD vs. LL

e

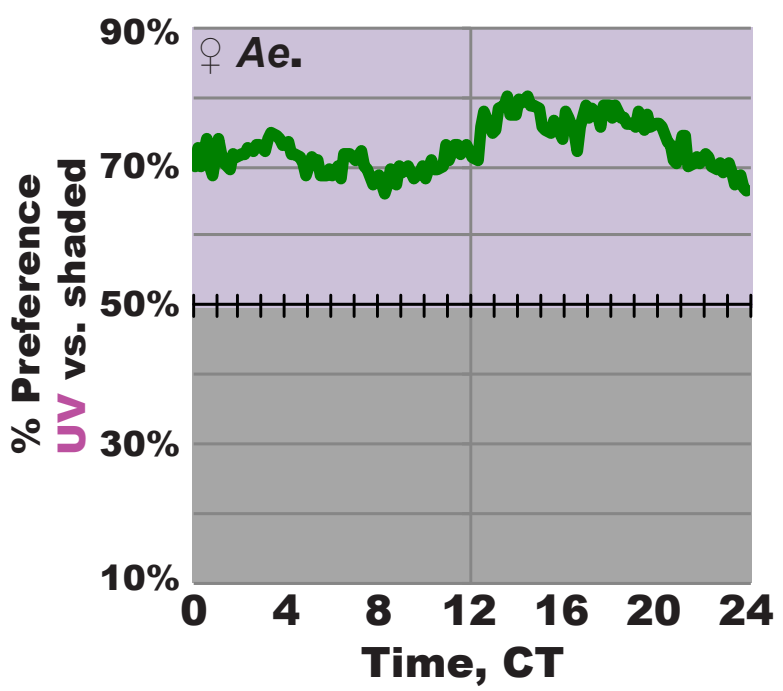

g

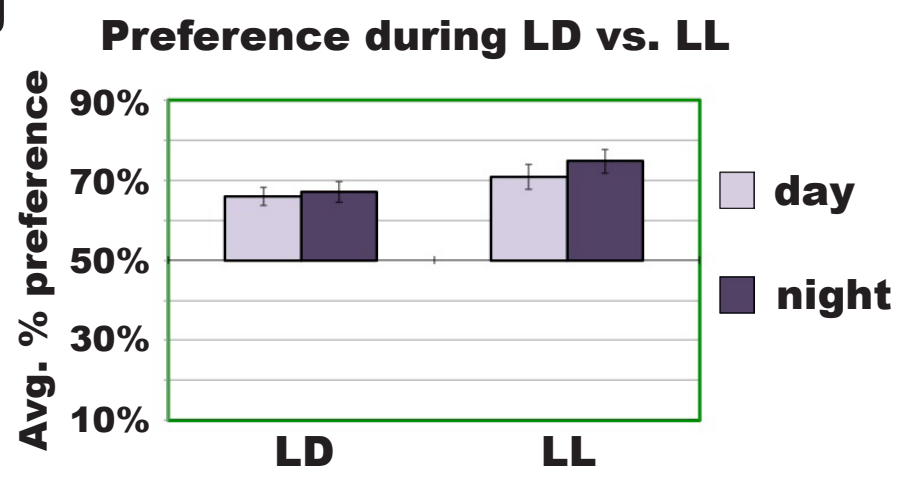

b

Anopheles coluzzii

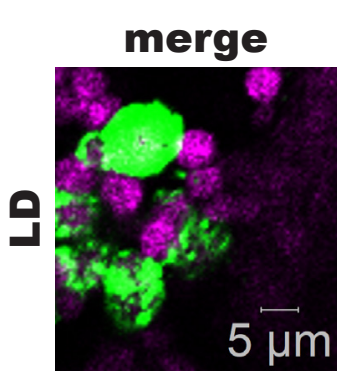

a-PDF
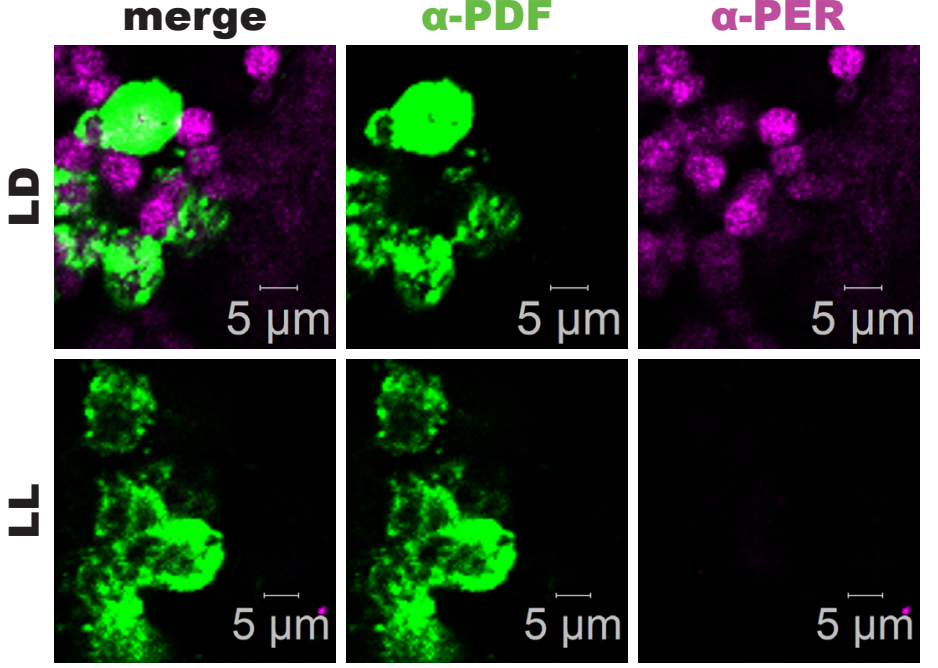

d

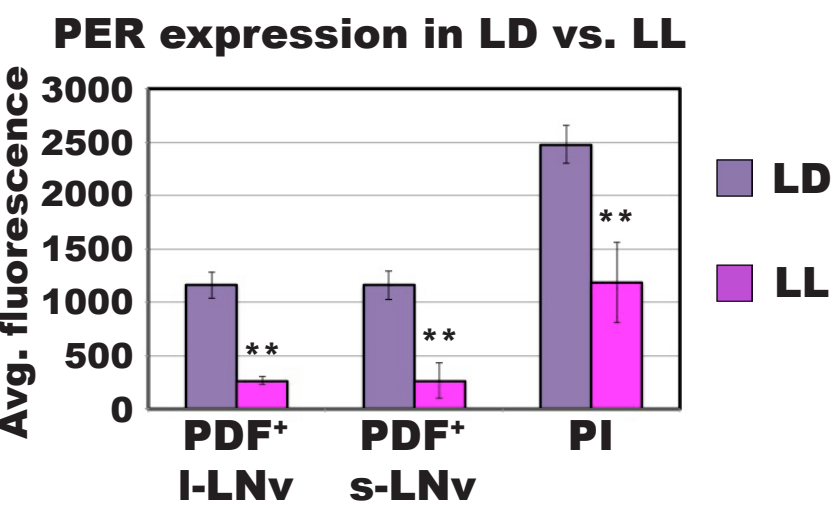

f

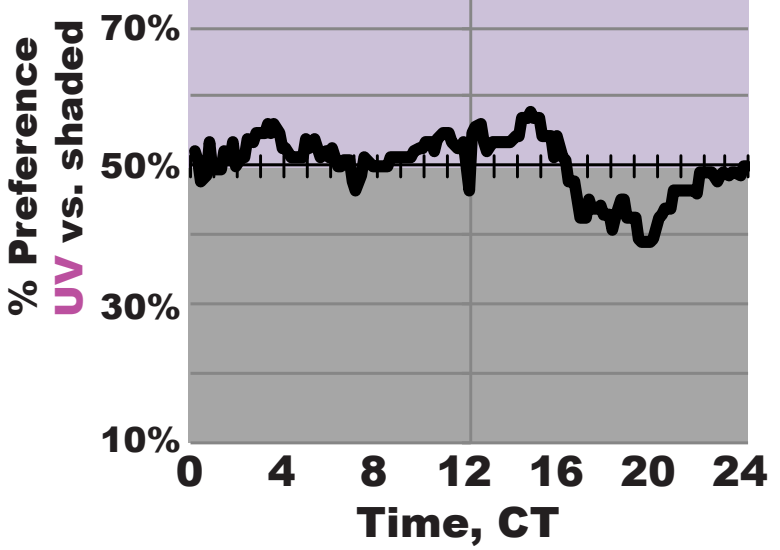

h

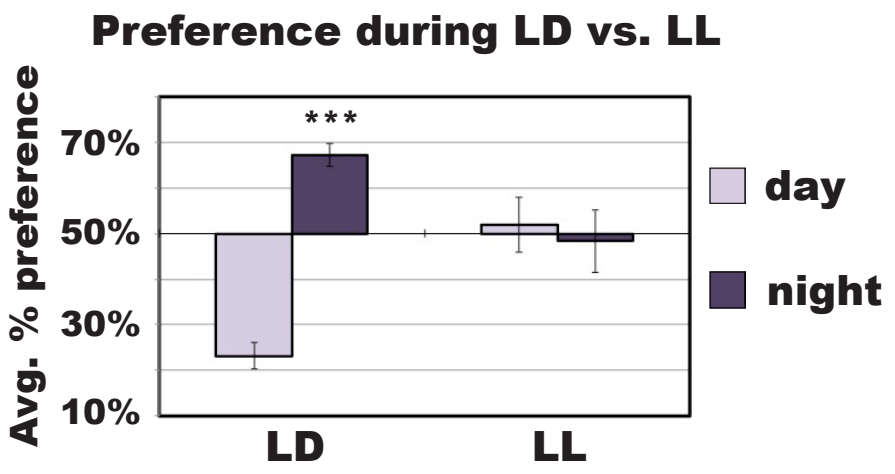

